\title{
In-Situ S/TEM of Li-Ion Batteries: Lithium Metal and Sn Anode Interfacial Processes
}

Katherine Jungjohann ${ }^{1}$, Katharine Harrison ${ }^{2}$, Subrahmanyam Goriparti ${ }^{1}$, William Mook ${ }^{1}$, Andrew Leenheer $^{3}$, and Kevin Zavadil ${ }^{2}$

1. Center for Integrated Nanotechnologies, Sandia National Laboratories, Albuquerque, USA.

2. Nanoscale Sciences Department, Sandia National Laboratories, Albuquerque, USA.

3. Microsystems Science \& Technology, Sandia National Laboratories, Albuquerque, USA.

Advanced portable energy storage technologies for electronic devices and transportation vehicles require an increase in the charge density to surpass the performance of state-of-the-art Li-ion batteries. Current Li-ion technologies are reliant on a graphite anode, which is inferior in theoretical capacity to other materials such as $\mathrm{Sn}$ or Li-metal. Battery degradation mechanisms such as material corrosion, selfdischarge, electrode pulverization, and metal dendrite formation, have limited the implementation of $\mathrm{Sn}$ and Li-metal anodes into commercial Li-ion cells. Our work has focused on the identification of nanoscale degradation mechanisms at electrode/electrolyte interfaces and the development of strategies to mitigate these limitations for the future implementation of $\mathrm{Sn}$ and Li-metal materials in advanced energy storage technologies.

Investigation of electrochemical ion transport mechanisms within the transmission electron microscope (TEM) at solid-liquid interfaces was developed in 2003 [1], and has since been advanced for customizable electrode layouts, multi-electrodes for several experiments within a single liquid cell, picoampere current control, constant liquid layer thickness over the entire window of the liquid cell [24], and elevated temperature control/sensing [5]. The Electrochemcial TEM Discovery Platform has demonstrated the ability to correlate the electrochemcial signatures to structural changes on/within a small $\left(c a .1 \mu \mathrm{m}^{2}\right)$ electrode at the nanoscale $[3,4]$. These platforms were customized to include relevant working electrodes of $\mathrm{Sn}$ or Li-metal vs. Li-containing counter and reference electrodes. All cells were prepared in an inert environment, with low levels of water to prevent artifacts in the electrochemcial behavior.

Integration of a Li-containing reference and counter electrode has enabled these electrochemcial TEM platforms to perform within the electrochemcial window that is relevant to bulk-scale battery testing. In comparison of bulk-scale batteries (such as coin cells) to the nanoscale structural and electrochemcial data from the TEM electrochemcial cell, we can better correlate the changes in performance of Li-metal and $\mathrm{Sn}$ electrodes to changes in the electrode/electrolyte interface. Protective films, artificial solidelectrolyte interphase layers, and chemically derived artificial solid-electrolyte interphase layers have been shown to greatly impact the Li plating/alloying behavior in these systems. The Li-metal electrodes were found to express an increased nucleation density and decreased self-discharge character when a $\mathrm{Li}_{\mathrm{x}} \mathrm{Al}_{\mathrm{y}} \mathrm{S}$ protective film was deposited on a current collector prior to Li plating [6]. In the Sn electrodes, the stability of the Sn within the electrolyte was greatly enhanced by allowing the electrode to sit for an extended period ( $c a .24$ hours) prior to Li alloying. This chemically induced solid electrolyte interphase that formed on the $\mathrm{Sn}$ behaved almost as well as an artificial film of $\mathrm{TiO}_{2}$, deposited by atomic-layer deposition. The ion transport mechanisms regarding the character of the solid/electrolyte interface for these systems will be discussed (Figure 1). Improvements to the electrochemcial TEM platform and possible artifacts induced by the electron beam during nanoscale imaging of these systems will be 
detailed. Electrochemical TEM testing of battery materials has provided nanoscale insights to electrochemcial and degradation mechanisms inherent to these systems that may have been overlooked during standard bulk-scale characterization techniques [7].

\section{References:}

[1] F M Ross, Science 350 (2015), p. 1490.

[2] A. J. Leenheer et al, J. Microelectromech. S. 99 (2015), p. 1061.

[3] A. J. Leenheer et al, ACS Nano 9 (2015), p. 4379.

[4] A. J. Leenheer et al, ACS Nano 10 (2016), p. 5670.

[5] A. J. Leenheer et al, Microsc. Microanal. 21 (S3) (2015), p. 1293.

[6] K. L. Harrison et al, ACS Nano 11 (2017), p. 11194.

[7] This work was performed, in part, at the Center for Integrated Nanotechnologies, an Office of Science User Facility operated for the U.S. Department of Energy (DOE) Office of Science. Sandia National Laboratories is a multi-mission laboratory managed and operated by National Technology and Engineering Solutions of Sandia, LLC., a wholly owned subsidiary of Honeywell International, Inc., for the U.S. Department of Energy's National Nuclear Security Administration under contract DE-NA0003525 .

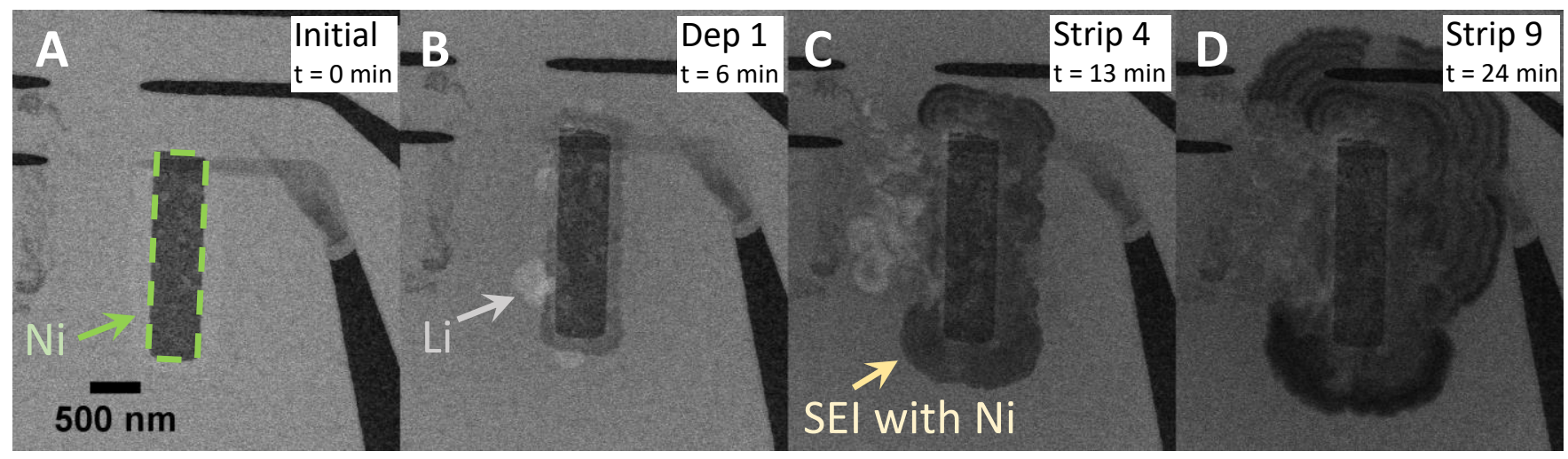

Figure 1. Li plating/stripping cycling on a Ni current collector, deposition from electrolyte [4 M lithium bis(trifluoromethanesulfonyl)imide in dimethoxyethane] at $6 \mathrm{~mA} / \mathrm{cm}^{2}$ for 60 seconds and stripping at 1.5 $\mathrm{mA} / \mathrm{cm}^{2}$ showing the artifact on the working electrode from corrosion of the unstable Ni counter electrode. A) The initial Ni working electrode of $500 \mathrm{~nm}$ x $2 \mu \mathrm{m}$ (in the center of the frame), connected by a $\mathrm{W}$ contact. B-D) Ni metal ions from the counter electrode plated on the working electrode along with Li metal (light intensity). This is shown as rings about the working electrode as the solid electrolyte interphase (SEI) layers grew during each deposition step along with plating of $\mathrm{Ni}$ metal atoms from the electrolyte. 